\title{
Material escrito para reducir la ansiedad en pacientes con dolor torácico agudo
}

Information sheets to reduce anxiety in patients with acute chest pain

Arnold J y col. BMJ 2009, feb 26. 338:b541

\section{Objetivo}

Determinar si la entrega de material escrito con información sobre su afección, a pacientes con dolor torácico agudo, reduce la ansiedad, mejora la calidad de vida y/o la satisfacción con la atención en los servicios de salud o modifica posteriores síntomas o acciones.

\section{Diseño, lugar y pacientes}

Estudio aleatorizado controlado y abierto realizado en la unidad de dolor torácico del departamento de emergencias de un hospital escuela de 1100 camas de Inglaterra. Participaron 700 pacientes de una edad promedio de 48,6 años (61,5\% varones) que estaban siendo estudiados por sospecha de síndrome coronario agudo en una unidad de dolor torácico. El seguimiento se realizó durante 15 meses.

\section{Intervención y medición de resultados principales} Se aleatorizó a los pacientes a dos grupos: control (349) e intervención (351). Fueron subdivididos en cuatro subgrupos cada uno según las características del dolor. Enfermeras especialistas en dolor torácico usaron marcadores cardíacos y pruebas de esfuerzo para seleccionar los casos. La intervención se llevo a cabo luego de que la evaluación fuera completada y el plan de tratamiento formulado. De acuerdo al diagnóstico se les entregó uno de los cuatro folletos informativos desarrollados por la Unidad de Ensayos Clínicos de Sheffield y luego de la aleatorización, ambos grupos recibieron un consejo verbal sobre dolor torácico. Después del asesoramiento, a los pacientes asignados al grupo de intervención se les dio información adecuada en un folleto para leer y llevar. Las enfermeras respondieron preguntas acerca de la información dada pero no sobre la información escrita.

Al mes y para evaluar el resultado primario (la ansiedad) se les envió por correo una escala de depresión y ansiedad ("Hospital Anxiety and Depression Scale" integrada por 14 preguntas) y para los resultados secundarios, un cuestionario de calidad de vida relacionada con la salud (el SF-36) un cuestionario de satisfacción ("Patient satisfaction survey") y otro acerca de la severidad y duración de los síntomas de dolor torácico y los cambios en el estilo de vida. De los 700 individuos incluidos en el estudio, 494 pacientes $(70,6 \%)$ respondieron los cuestionarios.

\section{Resultados principales}

Los puntajes para ansiedad y depresión fueron bajos en ambos grupos, resumiéndose los resultados positivos en la tabla 1. No hubo diferencias estadísticamente significativas para el resto de los resultados evaluados.

Tabla 1: resultados principales de la intervención.

\begin{tabular}{c|c|c|c|c}
\hline $\begin{array}{c}\text { Puntaje } \\
\text { ansiedad }\end{array}$ & $\begin{array}{c}\text { Grupo } \\
\text { control }\end{array}$ & $\begin{array}{c}\text { Grupo } \\
\text { intervención }\end{array}$ & $\begin{array}{c}\text { Diferencia } \\
\text { (IC95\%) }\end{array}$ & NNT \\
\hline & 8,63 & 7,61 & $1,02(0,2 \mathrm{a} 1,84)$ & 9 \\
\hline
\end{tabular}

0 a 7 puntos: ausencia de ansiedad; 8 a 10: síntomas leves; 11 a 14: síntomas moderados; 15 a 21: síntomas severos. NNT: número necesario a tratar.

\section{Conclusiones}

La provisión de información escrita a pacientes con dolor torácico reducirían la ansiedad y la depresión y mejorarían la salud mental y la percepción general del estado de salud, sin alterar la frecuencia o severidad de los síntomas de futuros dolores, los planes de cambio en el estilo de vida, la búsqueda de información, ni el plan de acción en respuesta a futuros dolores, ni la satisfacción del paciente con los cuidados.

Palabras claves: ansiedad, depresión, dolor torácico agudo, material escrito, información oral. Keywords: anxiety, depression, acute thoracic pain, written material, oral information. Fuentes de financiamiento: Health Foundation Leadership through Practice Award

\section{Comentario}

El dolor torácico es una de las causas más frecuente de consulta a los servicios de urgencias ${ }^{1,2}$. Arribar a un diagnóstico preciso es un verdadero desafío, especialmente cuando las características clínicas son atípicas y el electrocardiograma no presenta signos evidentes de isquemia miocárdica aguda ${ }^{3}$. Por otro lado, muchos pacientes presentan nuevos episodios, asociados a ansiedad y depresión ${ }^{4}$, lo que puede tener un importante impacto sobre su calidad de vida ${ }^{5}$. En este sentido, la entrega de material escrito constituye una buena herramienta para la comunicación6 y estudios anteriores habían arrojado

\section{resultados contradictorios ${ }^{7,8,9}$}

\section{Conclusiones del comentador}

Si bien se trata de una intervención inocua y de bajo costo, la investigación que hoy nos ocupa es un buen ejemplo de la identificación de diferencias estadísticamente significativas (algo más que un punto en una escala que va de 0 a 21) pero de escaso valor clínico, ya que de acuerdo a la herramienta utilizada, significó evitar un caso de ansiedad leve cada nueve pacientes sometidos a la intervención.

Esteban Noguez [ Policlínico Modelo de Cipolletti. ] enoguez@yahoo.com.ar

Recibido el 10/05/09 y aceptado el 16/07/09

Noguez E. Entrega de material escrito para minimizar la ansiedad en pacientes con dolor torácico agudo. Evid Act Pract Ambul. 12(3). 93. Jul-Sep. 2009. Comentado de: Arnold, J. Information sheets for patients with acute chest pain: randomised controlled trial. BMJ 2009, feb 26. 338 :b541.

\section{Referencia}

1.Mendoza B. Dolor torácico en el servicio de urgencias:"un reto por enfrentar" Rev. Col. Cardiol. 2003; 10: 455-464.

2.Goodacre $S$ y col. The health care burden of acute chest pain. Heart 2005; 91:229-30.

3.Botto F y col. Dolor precordial en la guardia: ¿un problema resuelto? Utilidad de la Unidad de Diagnóstico Intensivo. Rev. Argen Cardiol 2000; 68:193-202.

4.Fleet R y col. Panic disorder in emergency department chest pain patients: prevalence, comorbidity, suicidal ideation and physician recognition. Am J Med 1996;101:371-80. 5.Goodacre S y col. Psychological morbidity and health-related quality of life of patients assessed on a chest pain observation unit. Ann Emerg Med 2001;38:369-76.

6.Price $\mathrm{J}$ y col. Developing a rapid access chest pain clinic: qualitative studies of patients' needs and experiences. J Psychosom Res 2005;59:237-46.

7.Krishell S y col. Effect of emergency department information on patient satisfaction. Ann Emerg Med 1993;22: 568-72.

8.Kologlu M y col.Emergencydepartment information: does it affect patients' perception and satisfaction about the care given in an emergency department? Eur $\mathrm{J}$ Emerg Med 1999; 6:245-8.

1999; 6:245-8.
9un B y col. A patient education intervention does not improve satisfaction with emergency care. AnnEmerg Med 2004;44:378-83. 